\title{
Automated prediction of final infarct volume in patients with large-vessel occlusion acute ischemic stroke
}

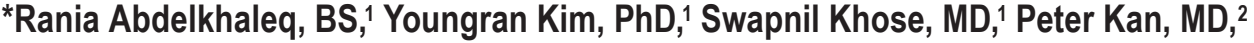 \\ Sergio Salazar-Marioni, MD, ${ }^{1}$ Luca Giancardo, PhD, ${ }^{3}$ and Sunil A. Sheth, MD' \\ 1Department of Neurology and ${ }^{3}$ Center for Precision Health, UTHealth School of Biomedical Informatics, UTHealth McGovern \\ Medical School, Houston; and '2Department of Neurosurgery, University of Texas Medical Branch, Galveston, Texas
}

\begin{abstract}
OBJECTIVE In patients with large-vessel occlusion (LVO) acute ischemic stroke (AIS), determinations of infarct size play a key role in the identification of candidates for endovascular stroke therapy (EVT). An accurate, automated method to quantify infarct at the time of presentation using widely available imaging modalities would improve screening for EVT. Here, the authors aimed to compare the performance of three measures of infarct core at presentation, including an automated method using machine learning.
\end{abstract}

METHODS Patients with LVO AIS who underwent successful EVT at four comprehensive stroke centers were identified. Patients were included if they underwent concurrent noncontrast head CT (NCHCT), CT angiography (CTA), and CT perfusion (CTP) with Rapid imaging at the time of presentation, and MRI 24 to 48 hours after reperfusion. NCHCT scans were analyzed using the Alberta Stroke Program Early CT Score (ASPECTS) graded by neuroradiology or neurology expert readers. CTA source images were analyzed using a previously described machine learning model named DeepSymNet (DSN). Final infarct volume (FIV) was determined from diffusion-weighted MRI sequences using manual segmentation. The primary outcome was the performance of the three infarct core measurements (NCHCT-ASPECTS, CTA with DSN, and CTP-Rapid) to predict FIV, which was measured using area under the receiver operating characteristic (ROC) curve (AUC) analysis.

RESULTS Among 76 patients with LVO AIS who underwent EVT and met inclusion criteria, the median age was 67 years (IQR 54-76 years), 45\% were female, and 37\% were White. The median National Institutes of Health Stroke Scale score was 16 (IQR 12-22), and the median NCHCT-ASPECTS on presentation was 8 (IQR 7-8). The median time between when the patient was last known to be well and arrival was 156 minutes (IQR 73-303 minutes), and between NCHCT/CTA/CTP to groin puncture was 73 minutes (IQR 54-81 minutes). The AUC was obtained at three different cutoff points: $10 \mathrm{ml}, 30 \mathrm{ml}$, and $50 \mathrm{ml}$ FIV. At the 50 -ml FIV cutoff, the AUC of ASPECTS was 0.74; of CTP core volume, 0.72; and of DSN, 0.82. Differences in AUCs for the three predictors were not significant for the three FIV cutoffs.

CONCLUSIONS In a cohort of patients with LVO AIS in whom reperfusion was achieved, determinations of infarct core at presentation by NCHCT-ASPECTS and a machine learning model analyzing CTA source images were equivalent to CTP in predicting FIV. These findings have suggested that the information to accurately predict infarct core in patients with LVO AIS was present in conventional imaging modalities (NCHCT and CTA) and accessible by machine learning methods.

https://thejns.org/doi/abs/10.3171/2021.4.FOCUS21134

KEYWORDS computed tomography; machine learning; CT perfusion; ischemic stroke; cerebrovascular disease/stroke

ABBREVIATIONS AIS = acute ischemic stroke; ASPECTS = Alberta Stroke Program Early CT Score; AUC = area under the ROC curve; CTA = CT angiography; CTP = CT perfusion; DSN = DeepSymNet; EVT = endovascular stroke therapy; FIV = final infarct volume; IV tPA = intravenous tissue plasminogen activator; LVO = large-vessel occlusion; NCHCT = noncontrast head CT; NIHSS = National Institutes of Health Stroke Scale; ROC = receiver operating characteristic; TICI = Thrombolysis in Cerebral Infarction.

ACCOMPANYING EDITORIAL DOI: 10.3171/2021.4.FOCUS21263.

SUBMITTED March 1, 2021. ACCEPTED April 6, 2021.

INCLUDE WHEN CITING DOI: 10.3171/2021.4.FOCUS21134.

* L.G. and S.A.S. contributed equally to this work. 
$\mathrm{E}$ NDOVASCULAR stroke therapy (EVT) is a highly effective intervention in patients with large-vessel occlusion (LVO) acute ischemic stroke (AIS), and its effectiveness is largely dependent on the extent of irreversibly injured, or infarcted, tissue at the time of patient presentation. ${ }^{1}$ At present, the optimal method to determine infarct core at the time of presentation remains unknown. The Alberta Stroke Program Early CT Score (ASPECTS) has been developed to quantify the extent of ischemia using noncontrast head CT (NCHCT), which is nearly universally available. On the other hand, ASPECTS grading can be subject to high interrater variability and can require a higher level of expertise for accurate reading than is available in many lower-volume centers at which AIS is evaluated. ${ }^{2}$ CT perfusion (CTP), on the other hand, benefits from automated determinations of infarcted tissue and played a large role in recent clinical trials. CTP is less widely available, however, and in addition has been shown to under- and overestimate the infarct core. ${ }^{3-5} \mathrm{~A}$ methodology that can accurately detect infarcted tissue, in an automated fashion, using imaging techniques that are currently in widespread use at all hospitals that evaluate AIS, including lower-volume centers, would improve screening for EVT.

To this end, we previously developed a machine learning model called DeepSymNet (DSN) that successfully predicted infarct core, as compared with concurrently acquired CTP, using a much more widely available modality, CT angiography (CTA), in an automated fashion. ${ }^{6,7}$ In this study, we hypothesized that NCHCT-ASPECTS and CTA with DSN perform adequately well in identifying the infarct core relative to CTP with Rapid (iSchemaView, Inc.) in patients with LVO AIS. We compared the performance of these three modalities at predicting the final infarct volume (FIV) in patients who underwent successful EVT.

\section{Methods \\ Study Population}

The data that support the findings of this study are available from the corresponding author on reasonable request. Our study population consisted of a subset of the Practical Implementation of Mechanical Thrombectomy (PRIME) study. PRIME is a prospective observational cohort study examining all patients diagnosed with AIS or transient ischemic attack at 11 Joint Commission-certified stroke centers within the same health system across the Greater Houston area. For this study, we identified a subset of this population that presented to four of these hospitals, which are comprehensive stroke centers, with onsite CTP and EVT capabilities, between March 2016 and April 2019. Patients were included if they were diagnosed with anterior circulation LVO AIS and underwent an imaging workup that included simultaneous NCHCT, CTA, and CTP with Rapid postprocessing at the time of presentation. In all patients, CTA was used to assess the presence of LVO. LVO was defined as an occlusion of the intracranial internal carotid artery, $A_{1}$ or $A_{2}$ segments of the anterior cerebral artery, or $\mathrm{M}_{1}$ or $\mathrm{M}_{2}$ segments of the middle cerebral artery. Patients were also required to have undergone EVT with successful reperfusion (Thromboly- sis in Cerebral Infarction [TICI] grade of $2 b$ or 3 ), and to have had FIV imaging consisting of MRI at 24 to 48 hours after reperfusion. In all patients, NCHCT, CTA, and CTP were acquired concurrently, which was at the time of presentation to the hospital.

The study was reviewed and approved by the Committee for the Protection of Human Subjects at The University of Texas Health Science Center at Houston, and waiver of consent and Health Insurance Portability and Accountability Act (HIPAA) authorization was granted.

\section{Measurements}

All study data were collected and managed using Research Electronic Data Capture (REDCap) tools. ${ }^{8,9}$ Quantification of ischemic core at the time of presentation was made by three methods. First, ASPECTS was determined from NCHCT using a neuroradiology report, or if it was not available, by an expert reviewer with experience in AIS imaging determinations. Second, CTP studies were postprocessed using Rapid. Finally, CTA source images were analyzed using DSN-v2 (see below). We defined FIV on diffusion-weighted MRI performed within 72 hours after successful EVT, consistent with prior studies.,10-12 FIV was measured utilizing the manual region of interest measurements (Horos, https://horosproject.org).

\section{DeepSymNet-v2}

DSN-v2 is a machine learning algorithm based on a 3D convolutional neural network that utilizes brain hemisphere symmetry information in order to learn imaging patterns that are predictive of outcomes related to stroke. In our previous works, we described the first iteration of the algorithm, DSN, which was used to detect LVO, estimate infarct core, and detect hemorrhagic stroke. . $^{6,113}$ In this study, we utilized a new version of the machine learning model, which has been improved by including a CTA-specific template, multiresolution pipeline, and skull stripping. The model was further improved by using symmetric and nonsymmetric paths, and by reducing the number of parameters by using more efficient building blocks, skip connections, and batch normalization. ${ }^{14,15}$

The algorithm for this machine learning model was trained and internally validated on an external CTA data set acquired from patients outside this analysis cohort, allowing for a meaningful estimation of the algorithm generalizability and the ability to fine-tune its architecture. At training time, the algorithm used a binary variable indicating a large $(>50 \mathrm{ml})$ or small $(<50 \mathrm{ml})$ infarct core as a target, which was computed from the CTP image and the Rapid analysis software. At inference time (i.e., when the algorithm was evaluated in this analysis) the algorithm generated a real number ranging from 0 to 1 in indicating the noncalibrated likelihood of having a small infarct core, with values closer to 1 more indicative of a smaller core.

\section{Primary Outcome}

The primary outcome of this study was the performance of the three infarct core measures-NCHCT-ASPECTS, DSN-v2 model (using CTA), and CTP-Rapid-at predicting FIV in this cohort of patients with successful EVT. 
This outcome was measured using area under the receiver operating characteristic (ROC) curve (AUC) statistics at three FIV cutoffs $(10 \mathrm{ml}, 30 \mathrm{ml}$, and $50 \mathrm{ml})$. These cutoff values were chosen to show the performance of the algorithm at different core sizes. They were based on clinically relevant values while also ensuring we had sufficient data with our distribution of core sizes to perform the analysis. The $10-\mathrm{ml}$ cutoff was chosen as a lower value. The $30-\mathrm{ml}$ and $50-\mathrm{ml}$ cutoffs were chosen to be consistent with the core thresholds used in the DAWN trial. ${ }^{16}$

\section{Statistical Analysis}

For the description of the characteristics of the study population, percentages are reported for categorical variables, and medians (IQR) for continuous variables. All statistical tests were 2-sided, and conventional levels of significance $(\alpha=0.05)$ were used for interpretation. The model performance was measured using AUC statistics. The chi-square test was performed to test for significance between AUCs for the predictors. All data analyses were performed using Stata version 16 (StataCorp LLC) and Prism version 7 (GraphPad) software.

\section{Results}

Among 76 patients with LVO AIS who underwent EVT and met inclusion criteria, the median age was 67 years (IQR 54-76 years), $45 \%$ were female, and 37\% were White. Fifty percent of the patients had a history of hypertension, $21 \%$ had hyperlipidemia, and $12 \%$ had had a prior stroke. The median National Institutes of Health Stroke Scale (NIHSS) score was 16 (IQR 12-22), and 65\% of patients had a prestroke modified Rankin Scale score of 0. The median ASPECTS on presentation was 8 (IQR 7-8). The median time between when the patient was last known to be well and arrival was 156 minutes (IQR 73-303 minutes), and that between NCHCT/CTA/CTP to groin puncture was 73 minutes (IQR 54-81 minutes). Fifty-one percent of the patients received intravenous tissue plasminogen activator (IV tPA), and $25 \%$ achieved a TICI grade of $2 b$. More details on demographics and clinical characteristics can be found in Table 1 .

The distribution of infarct core predictions based on NCHCT-ASPECTS, CTP-Rapid, and DSN relative to FIV can be seen in Fig. 1. The performance of these three measures at predicting FIV can be seen in Fig. 2. The AUC was obtained at three different cutoff points: 10-ml, 30$\mathrm{ml}$, and 50-ml FIV. At the 10-ml FIV cutoff, the AUC of ASPECTS was 0.67; of CTP core volume, 0.63; and of DSN, 0.75. Differences in AUCs for the three predictors were not significant $(\mathrm{p}=0.11)$. At the $30-\mathrm{ml}$ FIV cutoff, the AUC of ASPECTS was 0.76; of CTP core volume, 0.73; and of DSN, 0.82. Differences in AUCs for the three predictors were not significant $(\mathrm{p}=0.36)$. At the $50-\mathrm{ml}$ FIV cutoff, the AUC of ASPECTS was 0.74; of CTP core volume, 0.72; and of DSN, 0.82. Differences in AUCs for the three predictors were not significant $(\mathrm{p}=0.44)$.

\section{Discussion}

In this study of different methods to measure infarct
TABLE 1. Demographic and clinical characteristics

\begin{tabular}{|c|c|}
\hline & All Patients $(n=76)$ \\
\hline Median age, yrs (IQR) & $67(54-76)$ \\
\hline Female sex, $\%$ & 44.7 \\
\hline \multicolumn{2}{|l|}{ Race, \% } \\
\hline White & 36.8 \\
\hline Black & 18.42 \\
\hline Asian & 5.26 \\
\hline Other & 39.47 \\
\hline \multicolumn{2}{|l|}{ Ethnicity, \% } \\
\hline Hispanic & 14.4 \\
\hline \multicolumn{2}{|l|}{ PMHx, \% } \\
\hline Prior stroke & 11.8 \\
\hline Hyperlipidemia & 21 \\
\hline Hypertension & 50 \\
\hline Diabetes & 22.3 \\
\hline Smoking & 10.5 \\
\hline \multicolumn{2}{|l|}{ Prestroke mRS score, $\%$} \\
\hline $0-2$ & 82.9 \\
\hline $3-5$ & 17.1 \\
\hline Median NIHSS score (IQR) & $16(12-22)$ \\
\hline Median baseline ASPECTS (IQR) & $8(7-8)$ \\
\hline Median Rapid ischemic core vol (IQR) & $4.5(0-21.5)$ \\
\hline Median FIV (IQR) & $11.39(2-37.5)$ \\
\hline \multicolumn{2}{|l|}{ Median time interval (IQR), mins } \\
\hline LKW to arrival & $156(73-303)$ \\
\hline CT to GP & $73(54-81)$ \\
\hline CT to recanalization & $111(100-126.5)$ \\
\hline \multicolumn{2}{|l|}{ Target occlusion site, $\%$} \\
\hline ICA & 13.3 \\
\hline $\mathrm{M}_{1}$ & 66.6 \\
\hline $\mathrm{M}_{2}$ & 16 \\
\hline Other & 4 \\
\hline Occlusion laterality It hemisphere, \% & 55.26 \\
\hline IV tPA, \% & 51 \\
\hline \multicolumn{2}{|l|}{ Final $\mathrm{TICl}$ grade, $\%$} \\
\hline $2 b$ & 25.3 \\
\hline 3 & 74.6 \\
\hline
\end{tabular}

core at the time of presentation in patients with LVO AIS, all three measurements showed good performance at predicting FIV in patients with recanalization. Our automated machine learning model analyzing CTA source images showed superior performance relative to NCHCTASPECTS and CTP-Rapid across the range of FIV sizes, although this difference was not statistically significant. The performances of all three models improved with identification of larger FIVs.

We chose to use FIV in patients with successful recanalization as the gold standard for infarct at presentation because of the known inaccuracies of other methods, par- 

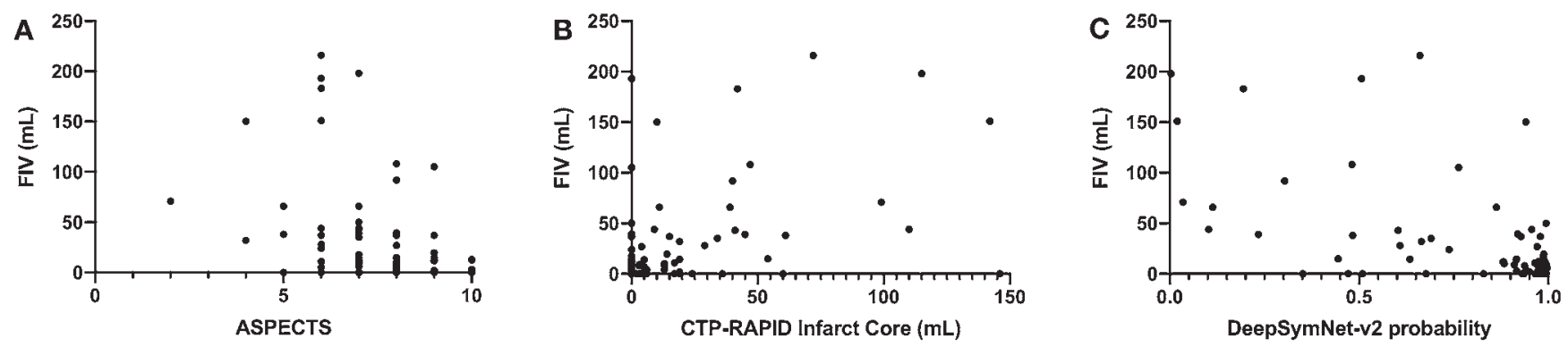

FIG. 1. Distribution of FIVs against three measurements of infarct core at presentation. Scatterplots showing the distribution of FIV versus ASPECTS $(\mathbf{A})$, CTP-Rapid infarct core $(\mathbf{B})$, and DSN $(\mathbf{C})$ probability on imaging performed at the time of patient presentation.

ticularly in the earlier time windows. ${ }^{4}$ There are, however, some limitations to this approach, as infarct volumes may grow from the time of imaging to reperfusion, and possibly even afterwards. On the other hand, this technique is consistent with prior studies evaluating the performance of machine learning methods for infarct core detection at the time of presentation. ${ }^{10,17,18}$ Furthermore, testing imaging methods using FIV in reperfused patients may have another advantage, as it measures the ability of the imaging methods to answer a clinically relevant question: if rapid reperfusion is achievable in this patient, what will be the expected outcome?

In the current study, we found that NCHCT-ASPECTS and DSN using CTA images performed equivalently to CTP-Rapid across a range of infarct sizes. These two imaging modalities, NCHCT and CTA, are far more widely available than CTP. Of these three methods, DSN boasts the advantage of being automated, thus requiring no expert input. In addition, the algorithm can be run on CTA source images in less than 1 minute. Given the known wide interrater reliability of NCHCT-ASPECTS, an automated approach to identify infarct is preferable, although recent advances in automated ASPECTS are improving in performance. ${ }^{10}$ In this cohort, the addition of CTP along with NCHCT and CTA did not add prognostic value to the imaging outcome.
Our study has several limitations. As mentioned above, it is possible that the infarct volume could grow even after the $<72$-hour MRI time point used here to define FIV. On the other hand, this time point has been used in many prior studies and has been shown to correlate closely with the 90-day clinical outcome. ${ }^{19}$ Furthermore, because we limited our cohort to patients who underwent EVT, the proportion of patients with very large infarcts was relatively small. While our findings would certainly benefit from validation in another data set, we did observe improved performance of ASPECTS and DSN with larger FIVs. Finally, our cohort contained primarily patients evaluated within the first 6 hours after last known well, and it is possible that these findings would not be replicated in patients who present in a later time window. Prior studies, on the other hand, have shown improved performance of NCHCT-ASPECTS with increasing time after last known well. ${ }^{20}$

\section{Conclusions}

In a cohort of patients with LVO AIS in whom reperfusion was achieved, determinations of infarct core at presentation by NCHCT-ASPECTS and a machine learning model analyzing CTA source images were equivalent to CTP in predicting FIV. The machine learning model
A

$\mathrm{FIV}<10 \mathrm{ml}$

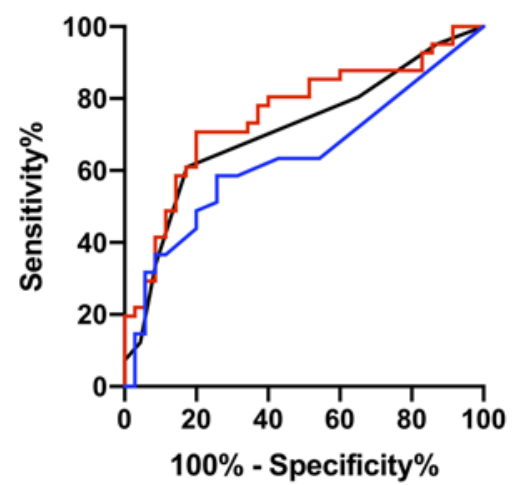

B

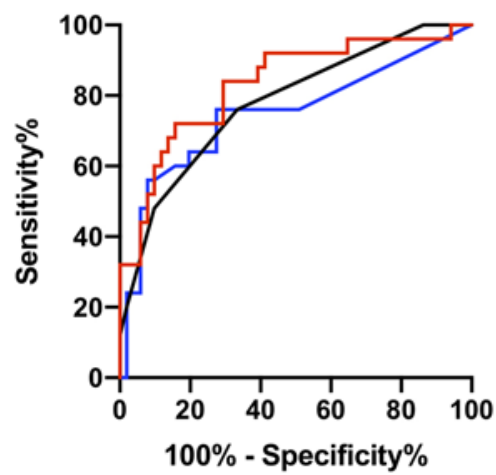

C

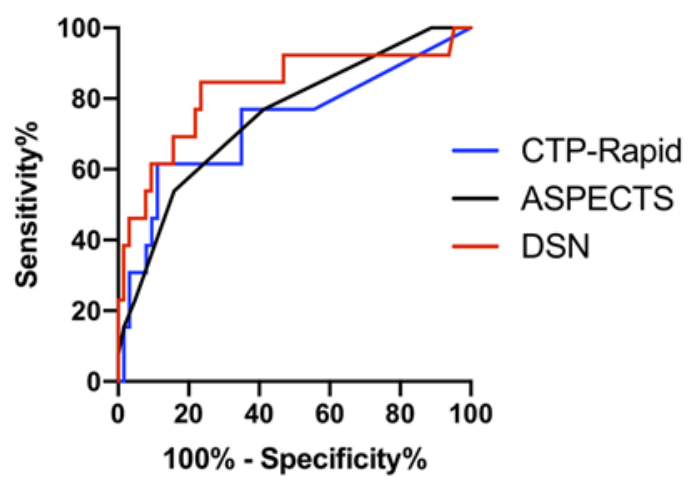

FIG. 2. Performance of three infarct core measurement techniques at presentation to predict FIV. AUCs at three FIV cutoffs of 10 $\mathrm{ml}(\mathbf{A}), 30 \mathrm{ml}(\mathbf{B})$, and $50 \mathrm{ml}(\mathbf{C})$ demonstrating the performance of three measures of infarct core at predicting FIV using imaging performed at the time of presentation, prior to EVT. 
demonstrated superior performance, but this difference was not statistically significant. These findings suggest that the information to accurately predict infarct core in patients with LVO AIS is present in conventional imaging modalities (NCHCT and CTA) and accessible by machine learning methods.

\section{References}

1. Goyal M, Menon BK, van Zwam WH, et al. Endovascular thrombectomy after large-vessel ischaemic stroke: a metaanalysis of individual patient data from five randomised trials. Lancet. 2016;387(10029):1723-1731.

2. Gupta AC, Schaefer PW, Chaudhry ZA, et al. Interobserver reliability of baseline noncontrast CT Alberta Stroke Program Early CT Score for intra-arterial stroke treatment selection. AJNR Am J Neuroradiol. 2012;33(6):1046-1049.

3. Copen WA, Yoo AJ, Rost NS, et al. In patients with suspected acute stroke, CT perfusion-based cerebral blood flow maps cannot substitute for DWI in measuring the ischemic core. PLoS One. 2017;12(11):e0188891.

4. Boned S, Padroni M, Rubiera M, et al. Admission CT perfusion may overestimate initial infarct core: the ghost infarct core concept. J Neurointerv Surg. 2017;9(1):66-69.

5. Geuskens RREG, Borst J, Lucas M, et al. Characteristics of misclassified CT perfusion ischemic core in patients with acute ischemic stroke. PLoS One. 2015;10(11):e0141571.

6. Barman A, Inam ME, Lee $S$, et al. Determining ischemic stroke from CT-angiography imaging using symmetrysensitive convolutional networks. In: 2019 IEEE 16th International Symposium on Biomedical Imaging (ISBI 2019). IEEE; 2019:1873-1877.

7. Sheth SA, Lopez-Rivera V, Barman A, et al. Machine learning-enabled automated determination of acute ischemic core from computed tomography angiography. Stroke. 2019; 50(11):3093-3100

8. Harris PA, Taylor R, Thielke R, et al. Research electronic data capture (REDCap) - a metadata-driven methodology and workflow process for providing translational research informatics support. J Biomed Inform. 2009;42(2):377-381.

9. Harris PA, Taylor R, Minor BL, et al. The REDCap Consortium: building an international community of software platform partners. J Biomed Inform. 2019;95:103208.

10. Bouslama M, Ravindran K, Harston G, et al. Noncontrast computed tomography e-stroke infarct volume is similar to RAPID computed tomography perfusion in estimating postreperfusion infarct volumes. Stroke. 2021;52(2):634-641.

11. Simonsen CZ, Yoo AJ, Sørensen LH, et al. Effect of general anesthesia and conscious sedation during endovascular therapy on infarct growth and clinical outcomes in acute ischemic stroke: a randomized clinical trial. JAMA Neurol. 2018;75(4): 470-477.

12. Yoo J, Choi JW, Lee SJ, et al. Ischemic diffusion lesion reversal after endovascular treatment. Stroke. 2019;50(6): 1504-1509.
13. Barman A, Lopez-Rivera V, Lee S, et al. Combining symmetric and standard deep convolutional representations for detecting brain hemorrhage. In: Medical Imaging 2020: Computer-Aided Diagnosis. Vol 11314. International Society for Optics and Photonics; 2020:113140D.

14. Huang G, Liu S, van der Maaten L, Weinberger KQ. CondenseNet: an efficient DenseNet using learned group convolutions. In: 2018 IEEE/CVF Conference on Computer Vision and Pattern Recognition. IEEE; 2018:2752-2761. Accessed May 10, 2021. https://ieeexplore.ieee.org/document/8578389

15. Ioffe S, Szegedy C. Batch normalization: accelerating deep network training by reducing internal covariate shift. Proc Mach Learn Res. 2015;37:448-456.

16. Nogueira RG, Jadhav AP, Haussen DC, et al. Thrombectomy 6 to 24 hours after stroke with a mismatch between deficit and infarct. N Engl J Med. 2018;378(1):11-21.

17. Austein F, Riedel C, Kerby T, et al. Comparison of perfusion CT software to predict the final infarct volume after thrombectomy. Stroke. 2016;47(9):2311-2317.

18. Robben D, Boers AMM, Marquering HA, et al. Prediction of final infarct volume from native CT perfusion and treatment parameters using deep learning. Med Image Anal. 2020;59: 101589.

19. Yoo AJ, Chaudhry ZA, Nogueira RG, et al. Infarct volume is a pivotal biomarker after intra-arterial stroke therapy. Stroke. 2012;43(5):1323-1330.

20. Nannoni S, Ricciardi F, Strambo D, et al. Correlation between ASPECTS and core volume on CT perfusion: impact of time since stroke onset and presence of large-vessel occlusion. AJNR Am J Neuroradiol. 2021;42(3):422-428.

\section{Disclosures}

Dr. Sheth reports grant funding from the National Institutes of Health, American Academy of Neurology, and Society for Vascular and Interventional Neurology.

\section{Author Contributions}

Conception and design: Sheth, Abdelkhaleq, Kim, Giancardo. Acquisition of data: Giancardo. Analysis and interpretation of data: Sheth, Abdelkhaleq, Giancardo. Drafting the article: Sheth, Abdelkhaleq, Kim, Khose, Salazar-Marioni, Giancardo. Critically revising the article: all authors. Reviewed submitted version of manuscript: all authors. Approved the final version of the manuscript on behalf of all authors: Sheth. Statistical analysis: Sheth, Kim. Administrative/technical/material support: Sheth. Study supervision: Sheth, Giancardo.

\section{Correspondence}

Sunil A. Sheth: UTHealth McGovern Medical School, Houston, TX.ssheth@post.harvard.edu. 